Jeremy A. Hirota ${ }^{1}$, Chris Carlsten ${ }^{1}$, Mohsen Sadatsafavi ${ }^{1}$, Gilaad Kaplan ${ }^{2}$ and Simon A. Hirota ${ }^{3}$

${ }^{1}$ Division of Respiratory Medicine, Dept of Medicine, Gordon and Leslie Diamond Health Care Centre, Vancouver, BC, Canada. ${ }^{2}$ Snyder Institute for Chronic Diseases and Institute of Public Health, Depts of Medicine and Community Health Sciences, Calgary, AB, Canada. ${ }^{3}$ Dept of Physiology and Pharmacology, Dept of Immunology, Microbiology and Infectious Diseases, Health Sciences Centre, Calgary, AB, Canada.

Correspondence: Jeremy A. Hirota, Division of Respiratory Medicine, Dept of Medicine, Gordon and Leslie Diamond Health Care Centre, 7th Floor, 2775 Laurel Street, Vancouver, BC, V5Z 1M9, Canada. E-mail: jhirota@mail.ubc.ca

Received: Jan 102015 | Accepted: Jan 252015

Conflict of interest: None declared.

\title{
References
}

1 Brassard P, Vutcovici M, Ernst P, et al. Increased incidence of inflammatory bowel disease in Quebec residents with airway diseases. Eur Respir J 2015; 45: 962-968.

2 Rutten EP, Lenaerts K, Buurman WA, et al. Disturbed intestinal integrity in patients with COPD: effects of activities of daily living. Chest 2014; 145: 245-252.

3 Tobin MV, Logan RF, Langman MJ, et al. Cigarette smoking and inflammatory bowel disease. Gastroenterology 1987; 93: 316-321.

4 Sava F, Carlsten C. Respiratory health effects of ambient air pollution: an update. Clin Chest Med 2012; 33: 759-769.

5 Kaplan GG, Hubbard J, Korzenik J, et al. The inflammatory bowel diseases and ambient air pollution: a novel association. Am J Gastroenterol 2010; 105: 2412-2419.

6 Hirota JA, Hirota SA, Warner SM, et al. The airway epithelium nucleotide-binding domain and leucine-rich repeat protein 3 inflammasome is activated by urban particulate matter. J Allergy Clin Immunol 2012; 129: $1116-1125$

7 Kish L, Hotte N, Kaplan GG, et al. Environmental particulate matter induces murine intestinal inflammatory responses and alters the gut microbiome. PLoS One 2013; 8: e62220.

8 Keely S, Talley NJ, Hansbro PM. Pulmonary-intestinal cross-talk in mucosal inflammatory disease. Mucosal Immunol 2012; 5: 7-18.

9 Hirota SA, Ng J, Lueng A, et al. NLRP3 inflammasome plays a key role in the regulation of intestinal homeostasis. Inflamm Bowel Dis 2011; 17: 1359-1372.

10 Birger N, Gould T, Stewart J, et al. The Air Pollution Exposure Laboratory (APEL) for controlled human exposure to diesel exhaust and other inhalants: characterization and comparison to existing facilities. Inhal Toxicol 2011; 23: 219-225.

\section{Bedaquiline: finding the pores on the pot}

To the Editor:

Salfinger and Migliori [1] discussed some important issues of bedaquiline, the most promising drug for treating multidrug-resistant (MDR) tuberculosis (TB) in the early twenty-first century. I am afraid of some issues that could be detrimental to the future of TB control. Bedaquiline should be used with caution, bacause of some methodological flaws in a few of the published landmark studies. In one study, DiACON et al. [2], the authors, without doing drug-sensitivity testing on isoniazid and rifampicin (criteria of MDR-TB), firmly concluded the effectiveness of their new regimen on MDR-TB patients. By using a regimen containing bedaquiline for susceptible patients to the tested drug does not predict bedaquiline's effectiveness in MDR-TB patients, as the pharmacokinetics and probability of pharmacodynamic target attainment (area under the time curve minimum inhibitory concentration ratio) are likely to be altered in MDR patients [3].

In another recent study by DiACON et al. [4], the efficacy analyses were performed in the modified intention-to-treat (ITT) population and selectively excluded patients after randomisation in their study. Some of the participants received the drug-resistant treatment, including bedaquiline, without confirmation of drug resistance and some with negative mycobacterial cultures. There is no clear definition of what is modified ITT. Modified ITT analysis has potential bias, due to the inappropriate exclusion of patients after including them in the trial. Post-randomisation exclusions is treated as deviations from protocol, destined for biased effect and strongly associated with industry funding [5]. So the real outcome is likely to be different in pragmatic trials or in the study outside of the trial conditions. ITT remains the best method that preserves randomisation. Well designed, randomised control trials follow strictly to the 
ITT principle, which is the best method for preserving randomisation irrespective of completing treatment in the group they were originally allocated.

Yet again, after a decade, there is still no study, to the best of my knowledge, on the: 1) impact of bedaquiline on clinical failure, 2) rate of relapse or survival, and 3) clinical resolution of TB. Drug toxicity can lead to decrease adherence and compliances of bedaquiline containing regimens in realistic conditions.

0 @ERSpublications

We need a better drug to control drug resistant tuberculosis (MDR TB) http://ow.ly/MTcSu

Prasanta Raghab Mohapatra

Dept of Pulmonary Medicine, All India Institute of Medical Sciences, Bhubaneswar, India.

Correspondence: Prasanta Raghab Mohapatra, Dept of Pulmonary Medicine, All India Institute of Medical Sciences, Bhubaneswar-751019, India. E-mail: prmohapatra@hotmail.com

Received: Feb 092015 | Accepted: Feb 112015

Conflict of interest: None declared.

\section{References}

1 Salfinger M, Migliori GB. Bedaquiline: 10 years later, the drug susceptibility testing protocol is still pending. Eur Respir J 2015; 45: 317-321.

2 Diacon AH, Dawson R, von Groote-Bidlingmaier F, et al. 14-day bactericidal activity of PA-824, bedaquiline, pyrazinamide, and moxifloxacin combinations: a randomised trial. Lancet 2012; 380: 986-993.

3 Chigutsa E, Meredith S, Wiesner L, et al. Population pharmacokinetics and pharmacodynamics of ofloxacin in South African patients with multidrug-resistant tuberculosis. Antimicrob Agents Chemother 2012; 56: 3857-3863.

4 Diacon AH, Pym A, Grobusch MP, et al. Multidrug-resistant tuberculosis and culture conversion with bedaquiline. N Engl J Med 2014; 371: 723-732.

5 Montedori A, Bonacini MI, Casazza G, et al. Modified versus standard intention-to-treat reporting: are there differences in methodological quality, sponsorship, and findings in randomized trials? A cross-sectional study. Trials 2011; 12: 58.

From the authors:

We read with interest the correspondence by Prasanta Raghab Mohapatra commenting on our editorial [1], which was aimed at discussing the absence of adequate drug susceptibility protocol for the new drug bedaquiline.

Prasanta Raghab Mohapatra raised interesting comments on the limitation of the trials presently available on bedaquiline and on the possible consequences for the patients.

With reference to the article by DiACON et al. [2], the authors investigated new multiple-agent combinations over the first 14 days of treatment to assess their suitability for future development. In this prospective, randomised, early bactericidal activity (EBA) study, treatment-naive, drug-susceptible patients with uncomplicated pulmonary tuberculosis (TB) were enrolled. We concur with the authors that multi-agent EBA studies can contribute to reducing the time needed to develop new anti-TB regimens.

Drug safety considerations are of outmost concerns for patients participating in clinical trials. In the case of bedaquiline, the risk of cardiological toxicity (prolongation of the QT interval in the electrocardiogram) and the unexplained higher toxicity in the treatment arm of the trial [3], pose additional queries that are still unanswered.

This necessary caution explains also why the World Health Organization (WHO), in absence of evidence on the simultaneous use of bedaquiline and delamanid, does not recommend the prescribing of these two drugs together [4] (table 1). In addition, WHO has developed the Policy Implementation Package for the introduction of new TB drugs (PIP document), underlining the minimum requirements that countries should have in place in order to use the new drugs safely, and with a reasonable low probability of developing further drug resistance (table 1).

We agree with Prasanta Raghab Mohapatra that properly designed trials are necessary to answer the numerous clinical queries that are still pending (table 1) [4].

In addition, there should be banking of Mycobacterium tuberculosis complex strains from patients participating in clinical trials, especially in those in whom therapy failed. Furthermore, novel compounds 